\title{
Persistence, adherence, and medication-taking behavior in women with postmenopausal osteoporosis receiving denosumab in routine practice in Germany, Austria, Greece, and Belgium: 12-month results from a European non-interventional study
}

\author{
P. Hadji ${ }^{1}$ N. Papaioannou ${ }^{2}$ E. Gielen ${ }^{3}$ - M. Feudjo Tepie ${ }^{4}$ E. Ehang ${ }^{4}$. \\ I. Frieling ${ }^{5}$ P. Geusens ${ }^{6,7} \cdot$ P. Makras $^{8}$ • H. Resch ${ }^{9} \cdot$ G. Möller ${ }^{10}$ • \\ L. Kalouche-Khalil ${ }^{11}$ • A. Fahrleitner-Pammer ${ }^{12}$
}

Received: 2 January 2015 / Accepted: 1 May 2015 / Published online: 28 May 2015

(C) The Author(s) 2015. This article is published with open access at Springerlink.com

\begin{abstract}
Summary Persistence with and adherence to osteoporosis therapy are critical for fracture reduction. This noninterventional study is evaluating medication-taking behavior of women with postmenopausal osteoporosis (PMO) receiving denosumab in Germany, Austria, Greece, and Belgium. Patients were representative of the PMO population and highly persistent with and adherent to denosumab at 12 months.

Introduction Persistence with and adherence to osteoporosis therapy are important for optimal treatment efficacy, namely fracture reduction. This ongoing, non-interventional study will evaluate medication-taking behavior of women with postmenopausal osteoporosis (PMO) receiving denosumab in routine practice in four European countries.
\end{abstract}

Electronic supplementary material The online version of this article (doi:10.1007/s00198-015-3164-4) contains supplementary material, which is available to authorized users.

P. Hadji

hadji.peyman@khnw.de

1 Department of Obstetrics and Gynecology, Krankenhaus Nordwest, Steinbacher Hohl 2-26, 60488 Frankfurt, Germany

2 Medical School, Laboratory of Research of Myoskeletal Diseases, KAT Hospital, University of Athens, Athens, Greece

3 Department of Geriatrics and the Centre for Metabolic Bone Diseases, UZ Leuven, Leuven, Belgium

4 Amgen Ltd, Uxbridge, UK

5 Osteoporosis Center, Hamburg, Germany
Methods The study enrolled women who had been prescribed subcutaneous denosumab (60 mg every 6 months) in accordance with prescribing information and local guidelines. Persistence was defined as receiving the subsequent injection within 6 months +8 weeks of the previous injection. Adherence was defined as receiving two consecutive injections within 6 months \pm 4 weeks of each other. Medication coverage ratio (MCR) was calculated using the time a patient was covered with denosumab, as assessed from prescription records. Treatment was assigned prior to and independently of enrollment; outcomes are recorded during routine practice. Results These planned 12-month interim analyses included data from 1500 patients from 141 sites. Mean age was 66.4 72.4 years, mean baseline total hip T-scores ranged from -2.0 to -2.1 and femoral neck T-scores from -2.2 to -2.6 , and

6 Department of Internal Medicine, Maastricht University Medical Centre, Maastricht, Netherlands

7 University Hasselt, Diepenbeek, Belgium

8 Department of Endocrinology and Diabetes, 251 Hellenic Airforce and VA General Hospital, Athens, Greece

9 St Vincent Hospital, Medical Department II, Academic Teaching Hospital of the Medical University of Vienna, Vienna, Austria

10 Practice for Preventive and Regenerative Medicine, Staufen im Breisgau, Germany

11 Amgen (Europe) GmbH, Zug, Switzerland

12 Department of Endocrinology and Metabolism, Medical University Graz, Graz, Austria 
30.7-62.1\% of patients had prior osteoporotic fracture. Persistence was $87.0-95.3 \%$, adherence $82.7-89.3 \%$, and MCR 91.3-95.4\%. In a univariate analysis, increased age, decreased mobility, and increased distance to the clinic were associated with significantly decreased persistence; parental history of hip fracture was associated with significantly increased persistence.

Conclusions These data extend the real-world evidence regarding persistence with and adherence to denosumab, both of which are critical for favorable clinical outcomes, including fracture risk reduction.

Keywords Adherence - Compliance - Denosumab · Non-interventional study · Osteoporosis · Persistence

\section{Introduction}

Persistence with and adherence to osteoporosis therapy are important factors in achieving successful treatment outcomes, in particular fracture reduction. Persistence is defined as taking medication for the prescribed treatment duration, and adherence is defined as taking the medication according to the dosing instructions [1]. Compared with ideal medicationtaking behavior, whereby recommended dosing regimens are followed, suboptimal persistence with and adherence to treatment of postmenopausal osteoporosis (PMO) increase a patient's risk of fracture by $17-64 \%$ [2-6].

Persistence and adherence rates for oral bisphosphonates for PMO are typically low, with up to two thirds of patients discontinuing treatment within 1 year of initiation $[2,7,8]$. Regimen complexity may be one barrier to persistence with and adherence to osteoporosis therapy: for example, patients prescribed oral bisphosphonates are required to take them on an empty stomach with water and then remain upright for at least $30 \mathrm{~min}$ [9]. Dosing frequency may also influence persistence and adherence, with several studies suggesting that oral bisphosphonate regimens requiring less frequent dosing result in better medication-taking behavior than do regimens with short-interval dosing [1, 10-14]. Furthermore, a meta-analysis found that patients preferred less frequent dosing regimens [13]. Long-acting injectable (LAI) therapies with less frequent dosing regimens may therefore improve persistence and adherence.

Data from studies in Germany, Hungary, and Spain have shown that persistence with osteoporosis medication after 1 year is considerably lower with oral dosing regimens (daily, weekly, or monthly) than with less frequent intravenous dosing regimens [13, 15-17]. However, even when using quarterly intravenous dosing schedules, between one half and two thirds of patients are not persistent 12 months after treatment initiation $[16,17]$. Nevertheless, improved quality of life has been observed in patients with previous fractures receiving yearly intravenous bisphosphonates compared with those receiving daily oral therapy [18], and several studies have shown that patients prefer annual intravenous infusions of zoledronic acid to weekly bisphosphonates [19]. While one study has shown that persistence is higher with yearly drugs than with daily, monthly, or quarterly drugs [17], further data on compliance and persistence for such injectable regimens are required [19].

Denosumab is a monoclonal antibody that selectively targets the RANK ligand, thereby inhibiting osteoclast formation, function, and survival [20]. In patients with osteoporosis, denosumab is administered by subcutaneous injection every 6 months [21]. In postmenopausal women with low bone mineral density (BMD), randomized, double-blind studies have demonstrated that denosumab significantly decreases markers of bone turnover and increases BMD, compared with the standard of care, weekly alendronate $[22,23]$. Compared with placebo, denosumab has been shown to reduce the risk of vertebral, non-vertebral, and hip fractures in women with PMO [24].

LAI treatments differ from oral treatments because they are usually administered by healthcare professionals rather than by patients themselves. Consequently, patients have little opportunity to deviate from the prescribing instructions, and the difference between persistence and adherence is less pronounced. Furthermore, the medication possession ratio (MPR; proportion of days for which a patient has an adequate supply of the medication over a defined time period) cannot be assessed for LAIs because the patient is covered therapeutically for a specific amount of time. Instead, the medication coverage ratio (MCR) can be calculated, which measures the percentage of days that the patient was covered by an LAI agent over a given time interval after receiving the injection. To ensure that women with PMO receive appropriate therapeutic intervention, it is imperative to assess persistence with and adherence to LAI therapy in a real-world setting and to explore optimal ways of evaluating these factors with respect to LAI treatments for osteoporosis, such as denosumab [25].

In order to gain a European perspective on the real-world use of denosumab in women with PMO, we are conducting a non-interventional study in Germany, Austria, Greece, and Belgium to investigate the medication-taking behavior of patients receiving denosumab treatment every 6 months in routine practice. Enrollment began in 2011 and recruitment has been completed. Here, we describe the results of pre-specified 12-month interim analyses.

\section{Methods}

\section{Study design}

These interim analyses are part of an ongoing, international, multicenter, prospective, non-interventional study that aims to assess 12-month persistence, adherence, and MCR in postmenopausal women receiving denosumab in routine practice 
in four European countries. These analyses will also report the incidence of adverse drug reactions (ADRs). Patients are being followed for 2 years after their first denosumab injection and are expected to receive subsequent injections every 6 months.

The study planned to enroll approximately 1500 patients across Germany, Austria, Greece, and Belgium ( $40 \%$ from Germany and $20 \%$ each from Austria, Greece, and Belgium) from 170 sites (60 in Germany, 30 each in Austria and Greece, and 50 in Belgium). Sites were selected to be representative of PMO care centers in each country.

To be eligible for inclusion in the study, patients must have been suitable for, and have been prescribed, subcutaneous denosumab $60 \mathrm{mg}$ every 6 months in accordance with the appropriate prescribing information (e.g., EU Summary of Product Characteristics or local equivalent) for the treatment of PMO and in accordance with national guidelines. Patients were excluded if they were currently enrolled, or had been enrolled in the past 6 months, in any other study involving another procedure, device, or drug or exhibited any disorder that the investigator felt might preclude the patient from providing informed consent.

To ensure that the recruitment strategy had as little impact on routine practice as possible, the decision to treat the patient with denosumab was made independently of, and before, enrollment in the study. After the initial denosumab injection, the informed consent and patient enrollment procedures were completed within 4 weeks; these steps could be completed on the same day, depending on clinic protocol and patient preference. Most baseline assessments were performed by the end of the fourth week following the first denosumab injection. For baseline assessments performed before the first injection, data obtained closest to the injection date were used. At enrollment, all patients completed the Morisky 8-Item Medication Adherence Scale (MMAS-8) questionnaire, which measures the likelihood of adherence on a scale of $0-8$ : high adherence, score of 8 ; medium adherence, score of 6-7; low adherence, score of less than 6 [26]. All other data were collected during routine practice. There was no requirement for additional tests such as dualenergy X-ray absorptiometry (DXA).

During this ongoing study, patients are not precluded from taking concomitant therapies that the investigators deem necessary, including calcium and vitamin D supplements. All information collected during routine practice was recorded using electronic case report forms. All occurrences of ADRs (as judged by the physician) were reported to the study sponsor (Amgen Inc.) by the physician.

\section{Analyses}

The interim analyses of baseline patient characteristics and 12month outcomes reported here were prospectively planned. They are based on the full analysis set, defined as all enrolled women who had received at least one injection of denosumab and who had a non-missing enrollment date.

Patient characteristics were summarized by country using descriptive statistics. Data are presented as number and percentage for categorical values and as mean and standard deviation (SD) for continuous variables. Persistence, adherence, and MCR at 12 months are reported by country as means and $95 \%$ confidence intervals (CIs). In addition, univariate logistic regression analysis was used to explore the association of each pre-specified baseline covariate with persistence at 12 months.

Persistence was defined as receipt of the subsequent injection within 6 months +8 weeks of the previous injection, and adherence as administration of the subsequent injection within 6 months \pm 4 weeks of the previous injection. Sensitivity analyses were conducted using alternative time windows of 6 months $+4,6$, and 12 weeks for persistence and 6 months $\pm 6,8$, and 12 weeks for adherence. The MCR was calculated using the percentage of time that a patient was covered by denosumab, as assessed from prescription records, and was based on the assumption that each injection of denosumab provides 6 months of medication coverage.

These interim analyses were carried out when all eligible women had had the opportunity to receive their second injection of denosumab and are still defined as persistent according to the longest time window after their initial denosumab injection (i.e., 6 months +12 weeks). Any data collected after the date of the second injection were not included. If a patient withdrew from the study before the second injection, the analyses included data collected before withdrawal. Future analyses are planned to ascertain persistence, adherence, and MCR at 24 months.

\section{Results}

\section{Study population}

A total of 1500 patients from 141 sites were included in the full analysis set: 599 women (48 sites) in Germany, 300 women (22 sites) in Austria, 300 women (27 sites) in Greece, and 301 women (44 sites) in Belgium.

\section{Physician demographics}

Physician specialties differed across the four countries. In Germany, the most common specialty was orthopedics (52.1\% of all physicians); in Austria, it was obstetrics/ gynecology (31.8\%); in Greece, the most common specialty was endocrinology (40.7\%), and in Belgium, it was family practice $(68.2 \%)$ (Table 1$)$. In most countries, the majority of physicians were male (81.5-89.6\% in Germany, Greece, and Belgium); in Austria, nearly half of the physicians were female $(45.5 \%)$. 
Table 1 Physician specialty according to country

\begin{tabular}{lllll}
\hline Specialty & Germany $(N=48)$ & Austria $(N=22)$ & Greece $(N=27)$ & Belgium $(N=44)$ \\
\hline Orthopedics $^{\mathrm{a}}$ & $25(52.1)$ & $3(13.6)$ & $9(33.3)$ & - \\
Endocrinology $^{\mathrm{a}}$ & $6(12.5)$ & $3(13.6)$ & $11(40.7)$ & - \\
Family practice $_{\text {Rheumatology }}$ & $2(4.2)$ & $4(18.2)$ & $0(0.0)$ & $30(68.2)$ \\
Rehabilitation medicine $^{\mathrm{b}}$ & $9(18.8)$ & $1(4.5)$ & $5(18.5)$ & $7(15.9)$ \\
Geriatrics $^{\mathrm{b}}$ & - & - & - & $1(2.3)$ \\
Obstetrics/gynecology $_{\text {Internal medicine }}^{\mathrm{a}}$ & $2(4.2)$ & - & - & $3(6.8)$ \\
Other $_{\text {Not available }}$ & $1(2.1)$ & $7(31.8)$ & $0(0.0)$ & $1(2.3)$ \\
Missing & $2(4.2)$ & $3(13.6)$ & $1(3.7)$ & - \\
\hline
\end{tabular}

Data are shown as $n(\%)$

$N$ number of physicians who enrolled women in each country, $n$ number of physicians reporting each specialty

${ }^{a}$ These specialties were not recorded in Belgium

${ }^{\mathrm{b}}$ These specialties were recorded only in Belgium

\section{Patient demographics, comorbidities, and medical history}

Baseline demographics (Table 2 and Supplementary Table 1) were generally similar across the countries. Mean age was lowest in Greece (66.4 years), compared with 71.0-72.4 years in Germany, Austria, and Belgium. Despite this age disparity, the time interval between diagnosis of PMO and the first denosumab injection was similar in all four countries (range of means, 6.0-6.5 years).

The most common comorbidities (as recorded by the treating physician) across the countries were hypertension (36.7-46.1\% of patients) and back pain (19.3-50.7\%), although the incidence of back pain was considerably lower in Greece (19.3\%) than in the other countries (Supplementary Table 1).

In all countries, a large proportion of patients had previously received therapy for PMO (81.3-88.8\%) (Table 2). Overall, patients had taken a mean of 2.0-2.6 different osteoporosis medications over the 5 years before enrollment (data not shown). Of the patients who had received prior osteoporosis medication, a large proportion had been prescribed oral bisphosphonates (40.2-62.8\%). Alendronate was the most common prior bisphosphonate therapy $(22.6-43.7 \%$ of patients), followed by risedronate (16.6-23.5\%) and ibandronate (7.0-20.2\%). One fifth of patients reported having stopped taking their prior osteoporosis medication (14.6$19.7 \%$ ) (data not shown).

While DXA assessment was not mandatory for study inclusion, the majority of patients had data from DXA assessment available at baseline (Germany, $87.8 \%$; Austria, 63.0\%; Greece $80.0 \%$; Belgium, $94.4 \%$ ). Mean baseline T-scores were low (range, -2.0 to -2.1 for total hip, -2.2 to -2.6 for femoral neck, and -2.2 to -2.8 for lumbar spine) (Table 2). Mean baseline hip, femoral neck, and lumbar spine T-scores were similar across the countries.
Self-reported medication-taking behavior for chronic health conditions was assessed at baseline using the MMAS8 questionnaire. The range of mean MMAS- 8 scores across the countries was 6.2-7.1. In all countries except Belgium, the majority of patients (for whom data were available) had a low or medium MMAS- 8 score for adherence to medications taken before the start of the study (Table 2); in Belgium, $37.5 \%$ of patients had a low or medium MMAS- 8 score.

The proportion of patients who had previously experienced an osteoporotic fracture varied by country; this was higher in Germany ( $62.1 \%$ of patients) than in Austria ( $40.7 \%$ ), Greece (30.7\%), and Belgium (50.2\%) (Table 2). Notably, prior vertebral fractures were considerably more common in Germany $(30.4 \%)$ than in the other three countries (range, 9.3-16.9 \%). Prior non-vertebral fractures were more common than prior vertebral fractures in all four countries.

In Austria, Greece, and Belgium, the most common reason cited by physicians for prescribing denosumab was that their patients had multiple risk factors for fracture (range, 35.3$54.8 \%$ of patients) (data not shown). In Germany, the most commonly cited reason was history of osteoporotic fracture (47.1\%). In general, a considerable proportion of patients were prescribed denosumab owing to failure of (26.6$44.7 \%$ of patients) or intolerance to (13.0-35.0\% of patients) their previous osteoporosis therapy (data not shown).

\section{Persistence, adherence, and medication coverage ratio at 12 months}

Across all four countries, 87.0-95.3\% of patients received a second denosumab injection within 6 months +8 weeks of the first injection and were therefore classified as persistent at 12 months (Fig. 1). Adherence at 12 months (i.e., the proportion of patients who had received a second denosumab 
Table 2 Baseline demographics, comorbidities, and medical history

\begin{tabular}{|c|c|c|c|c|}
\hline Characteristic & $\begin{array}{l}\text { Germany } \\
(N=599)\end{array}$ & $\begin{array}{l}\text { Austria } \\
(N=300)\end{array}$ & $\begin{array}{l}\text { Greece } \\
(N=300)\end{array}$ & $\begin{array}{l}\text { Belgium } \\
(N=301)\end{array}$ \\
\hline Age, years, mean (SD) & $72.4(8.6)$ & $71.0(9.5)$ & $66.4(9.3)$ & $71.2(10.4)$ \\
\hline Age at menopause, years, mean (SD) & $48(5.8)$ & $49.4(5.8)$ & $47.7(5.3)$ & $49.4(4.7)$ \\
\hline Time since PMO diagnosis, years, mean (SD) & $6.0(5.6)$ & $6.5(6.5)$ & $6.4(6.3)$ & $6.4(8.2)$ \\
\hline \multicolumn{5}{|l|}{ Smoking, $n(\%)$} \\
\hline Never & $427(71.3)$ & $212(70.7)$ & $228(76.0)$ & $236(78.4)$ \\
\hline Formerly & $52(8.7)$ & $40(13.3)$ & $24(8.0)$ & $32(10.6)$ \\
\hline Currently & $50(8.3)$ & $48(16.0)$ & $47(15.7)$ & $33(11.0)$ \\
\hline Missing & $70(11.7)$ & $0(0.0)$ & $1(0.3)$ & $0(0.0)$ \\
\hline Prior osteoporotic fracture, $n(\%)$ & $372(62.1)$ & $122(40.7)$ & $92(30.7)$ & $151(50.2)$ \\
\hline Vertebral & $182(30.4)$ & $28(9.3)$ & $34(11.3)$ & $51(16.9)$ \\
\hline Non-vertebral & $260(43.4)$ & $101(33.7)$ & $65(21.7)$ & $126(41.9)$ \\
\hline \multicolumn{5}{|l|}{ Parental history of hip fracture, $n(\%)$} \\
\hline Yes & $54(9.0)$ & $34(11.3)$ & $52(17.3)$ & $29(9.6)$ \\
\hline No & $272(45.4)$ & $197(65.7)$ & $192(64.0)$ & $188(62.5)$ \\
\hline Unknown & $273(45.6)$ & $69(23.0)$ & $55(18.3)$ & $84(27.9)$ \\
\hline Missing & $0(0.0)$ & $0(0.0)$ & $1(0.3)$ & $0(0.0)$ \\
\hline Prior PMO therapy, $n(\%)$ & $532(88.8)$ & $252(84.0)$ & $244(81.3)$ & $256(85.0)$ \\
\hline \multicolumn{5}{|l|}{ Baseline T-scores, mean (SD) } \\
\hline Total hip & $-2.0(0.8)$ & $-2.0(0.8)$ & $-2.0(0.9)$ & $-2.1(0.9)$ \\
\hline Femoral neck & $-2.3(0.8)$ & $-2.2(0.8)$ & $-2.6(0.8)$ & $-2.5(0.7)$ \\
\hline Lumbar spine & $-2.7(1.1)$ & $-2.8(0.9)$ & $-2.7(0.8)$ & $-2.2(1.3)$ \\
\hline \multicolumn{5}{|l|}{ MMAS- $8^{\mathrm{a}}$} \\
\hline Total adherence score, mean (SD) & $7.0(1.2)$ & $6.6(1.5)$ & $6.2(1.8)$ & $7.1(1.6)$ \\
\hline Low or medium adherence score, $n(\%)$ & $266(57.6)$ & $177(67.0)$ & $187(74.8)$ & $113(37.5)$ \\
\hline
\end{tabular}

MMAS-8 Morisky 8-Item Medication Adherence Scale, PMO postmenopausal osteoporosis, SD standard deviation

${ }^{\text {a }}$ Scores calculated from women who answered all questions in the MMAS- 8 questionnaire. Scores ranged from 0 to 8 , with high adherence represented by a score of 8 , medium adherence by a score of $6-7$, and low adherence by a score of less than 6

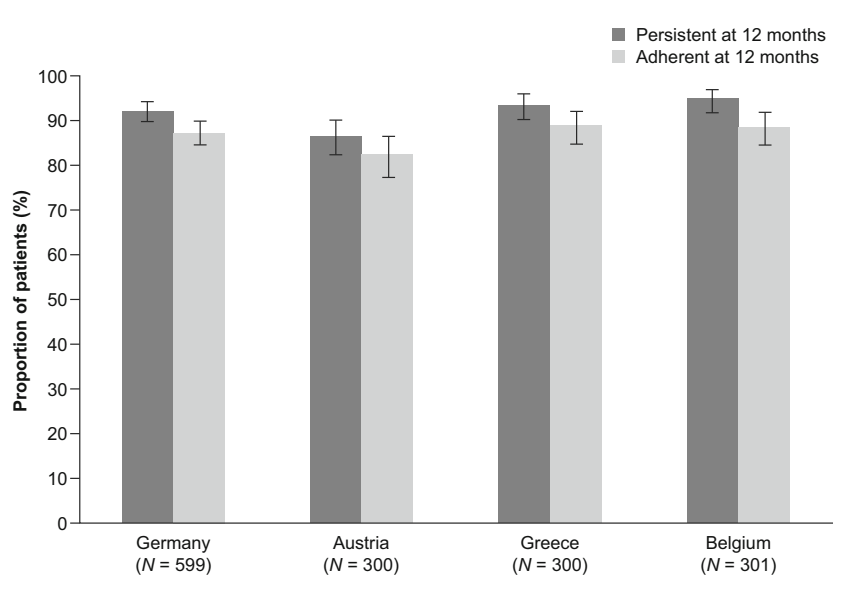

Fig. 1 Persistence with and adherence to denosumab at 12 months. Data are shown as percentage $\pm 95 \%$ confidence interval. Persistence was defined as receiving the subsequent injection within 6 months +8 weeks of the previous injection. Adherence was defined as receiving two consecutive injections within 6 months \pm 4 weeks of each other injection within 6 months \pm 4 weeks of the first injection) was 82.7-89.3 \% (Fig. 1). Mean MCR was 91.3-95.4 \% (Fig. 2).

Sensitivity analyses confirmed that, in all countries, persistence and adherence increased as the length of the permissible time window increased (Table 3). In Austria and Greece, patients who had at least one baseline DXA measurement at any skeletal site tended to have increased persistence compared with those without any DXA measurements (12-month persistence, 93.9 and $95.4 \%$ versus 64.3 and $66.7 \%$, respectively) (data not shown). In Germany and Belgium, there was no difference in persistence according to whether baseline DXA measurements had been taken (data not shown).

\section{Baseline factors associated with 12-month persistence}

Univariate analyses for all baseline covariates by country can be found in Supplementary Tables 2-5; variables deemed potentially clinically relevant are discussed herein. 


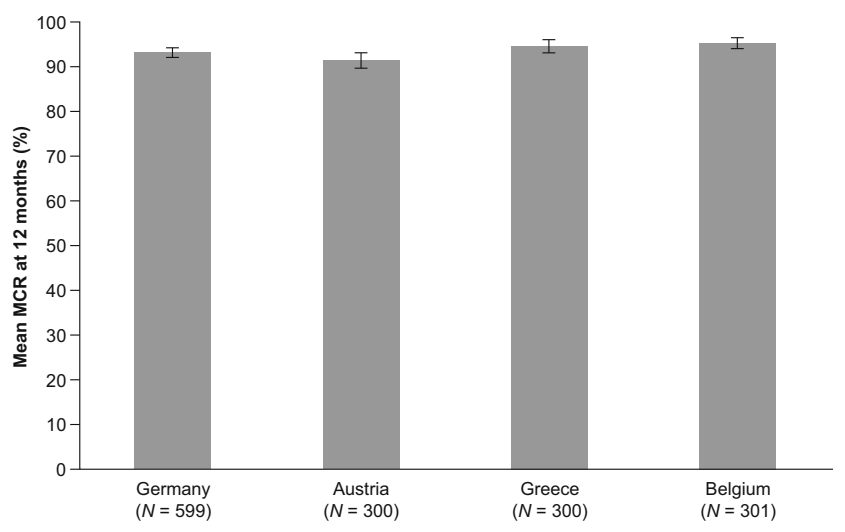

Fig. 2 Medication coverage ratio of denosumab at 12 months. Data are shown as mean percentage $\pm 95 \%$ confidence interval. The medication coverage ratio (MCR) was calculated as the percentage of days that a patient was covered by denosumab, as assessed from prescription records, and was based on the assumption that each injection of denosumab provides 6 months of medication coverage

The following baseline covariates did not influence persistence $(p>0.05)$ in any of the four countries: height loss, historical fracture, historical vertebral fracture, hospitalization for osteoporotic fracture prior to enrollment, current and former smoker status, femoral neck and lumbar spine T-scores, time between diagnosis of PMO and first denosumab injection, chronic medical conditions, SF-12 Physical and Mental Component Summary scores, MMAS-8 scores, number of comorbidities at baseline, highest education level achieved, living situation, marital status, academic or non-academic center, and calcium and/or vitamin D supplementation.

While no baseline covariates were found to have a statistically significant association with persistence in all four countries, there were some consistent trends. Across all countries, patients who reported that one of their parents had experienced a hip fracture and those aged younger than 75 years tended to have higher persistence than those who did not report a parental history of hip fracture and those aged 75 years or older, and statistical significance was reached for parental history of hip fracture in Austria $(p=0.0413)$ and for age in Greece $(p=0.0398)$. In Germany, Greece, and Belgium, patients whose traveling time to their treating clinic exceeded the median traveling time for their country tended to have lower persistence than those with a traveling time lower than or equal to the median, and statistical significance was reached in Belgium ( $p=0.0446$ [median traveling time, $10.0 \mathrm{~min}])$. In Germany, Austria, and Greece, patients experiencing one or more falls or occurrences of immobility during the 12 months prior to enrollment tended to have lower persistence than those with no falls or occurrences of immobility, and statistical significance for both covariates was reached in Austria ( $p=0.0011$ and 0.0074 , respectively).

There were some country-specific differences regarding which covariates influenced persistence. In Austria, three or more prescription medications at baseline, any prior exposure to PMO therapy, and exposure to PMO therapy in the 12 months prior to enrollment were associated with significantly higher persistence compared with fewer medications, having never been exposed to PMO therapy, and no exposure to PMO therapy in the 12 months prior to enrollment ( $p=0.0269$, 0.0293 , and 0.0374 , respectively). Patients enrolled at sites participating in the Prolong patient support program and those whose physician was an obstetrician/gynecologist had significantly higher persistence compared with those enrolled at sites not taking part in the Prolong program and those whose physician was not an obstetrician/gynecologist $(p<0.0001$ and 0.0025 , respectively). None of these trends were observed in the other countries. While experiencing two or more historical fractures was significantly associated with increased persistence in Austria $(p=0.0267)$, the opposite trend was observed in the other countries, which was statistically significant in Germany and Belgium ( $p=0.0462$ and 0.0314 , respectively).

In Germany, a history of discontinuation of prior PMO therapy was associated with significantly decreased persistence $(p=0.0241)$, and a similar but non-significant trend was observed in Austria. In Greece, this covariate was associated with significantly increased persistence $(p=0.0035)$, and a similar but non-significant trend was observed in Belgium.

\section{Safety}

Across the four countries, 1.7-5.0\% of patients reported ADRs (Table 4). The most commonly reported ADRs affected
Table 3 Sensitivity analysis of persistence and adherence at 12 months

\begin{tabular}{lllll}
\hline & Germany $(N=599)$ & Austria $(N=300)$ & Greece $(N=300)$ & Belgium $(N=301)$ \\
\hline $\begin{array}{l}\text { Persistence } \\
6 \text { months }+4 \text { weeks }\end{array}$ & $89.0(86.2-91.4)$ & $84.3(79.7-88.3)$ & $90.0(86.0-93.2)$ & $91.7(88.0-94.6)$ \\
6 months+6 weeks & $91.0(88.4-93.2)$ & $86.0(81.6-89.7)$ & $93.0(89.5-95.6)$ & $94.0(90.7-96.4)$ \\
6 months+12 weeks & $93.0(90.6-94.9)$ & $87.7(83.4-91.2)$ & $95.3(92.3-97.4)$ & $96.0(93.1-97.9)$ \\
Adherence & & & & \\
6 months \pm 6 weeks & $90.8(88.2-93.0)$ & $85.7(81.2-89.4)$ & $92.7(89.1-95.3)$ & $93.7(90.3-96.2)$ \\
6 months \pm 8 weeks & $92.5(90.1-94.5)$ & $87.0(82.7-90.6)$ & $93.7(90.3-96.1)$ & $95.3(92.3-97.4)$ \\
6 months \pm 12 weeks & $93.0(90.6-94.9)$ & $87.7(83.4-91.2)$ & $95.0(91.9-97.2)$ & $96.0(93.1-97.9)$ \\
\hline
\end{tabular}

Data are shown as percentage (95\% confidence interval) 
Table 4 Safety data at 12 months

\begin{tabular}{lcccc}
\hline Outcome & $\begin{array}{l}\text { Germany } \\
(N=599)\end{array}$ & $\begin{array}{l}\text { Austria } \\
(N=300)\end{array}$ & $\begin{array}{l}\text { Greece } \\
(N=300)\end{array}$ & $\begin{array}{l}\text { Belgium } \\
(N=301)\end{array}$ \\
\hline All adverse drug reactions & $30(5.0)$ & $8(2.7)$ & $8(2.7)$ & $5(1.7)$ \\
Leading to discontinuation of denosumab & $15(2.5)$ & $2(0.7)$ & $1(0.3)$ & $2(0.7)$ \\
Serious adverse drug reaction & $3(0.5)$ & $0(0.0)$ & $0(0.0)$ & $1(0.3)$ \\
Leading to death & $0(0.0)$ & $0(0.0)$ & $0(0.0)$ & $0(0.0)$ \\
Adjudicated positive ONJ & $2(0.3)$ & $0(0.0)$ & $0(0.0)$ & $0(0.0)$ \\
Osteoporotic fracture $^{\mathrm{a}}$ & $10(1.7)$ & $2(0.7)$ & $7(2.3)$ & $8(2.7)$ \\
Adjudicated positive for atypical femoral fracture & $0(0.0)$ & $0(0.0)$ & $0(0.0)$ & $0(0.0)$ \\
Delayed fracture healing & $0(0.0)$ & $0(0.0)$ & $0(0.0)$ & $0(0.0)$ \\
\hline
\end{tabular}

Data are shown as $n(\%)$

ONJ osteonecrosis of the jaw

${ }^{a}$ Osteoporotic fractures were defined as all fractures excluding those of the skull, facial bones, mandible, metacarpus, finger phalanges, toe phalanges, and cervical vertebrae and those not associated with known high trauma severity (fall from higher than the height of stool, chair, or first rung on a ladder or equivalent [ $>20 \mathrm{in}$.], or severe trauma other than a fall) and pathological fractures the musculoskeletal and connective tissue systems $(0.3-2.0 \%$ of patients; data not shown), for example, arthralgia, pain in extremity, back pain, or myalgia. Overall, $0.3 \%$ of patients reported serious ADRs, with no fatal events. The serious ADRs comprised two cases of osteonecrosis of the jaw (ONJ) (discussed below), one case of liver metastases diagnosed 10 months after denosumab initiation (in a patient with gall bladder cancer at enrollment) that resulted in discontinuation of denosumab, and one case of musculoskeletal pain. Overall, $0.7 \%$ of patients reported ADRs relating to skin and subcutaneous tissue disorders, including pruritus, erythe$\mathrm{ma}$, and rash. No cases of hypocalcemia or anaphylaxis were reported in any country. Twenty-seven women reported ADRs of osteoporotic fracture $(1.8 \%)$, including fractures of the lumbar spine, humerus, wrist, hip, ribs, and pelvis (data not shown). At the time of the interim analyses, there were no reports of atypical femoral fractures or delayed fracture healing (Table 4).

Two independently adjudicated cases of ONJ were reported, both in Germany (Table 4). Both individuals had risk factors for ONJ (previous bisphosphonate use, old age, or invasive dental procedures). One case resolved after approximately 1 year. As of the last follow-up report received from the treating physician (July 2013), the second case was ongoing.

\section{Discussion}

Results from these interim analyses show that persistence with denosumab at 12 months was consistently high in all four countries studied (87.0-95.3\%). Moreover, the persistence observed in the current study was at least 1.5-10-fold higher than the 12-month persistence from similar studies of bisphosphonates $(8.5-58 \%)[2,16,17]$. Adherence and
MCR were also high in all countries, and the safety results were consistent with those observed in previous studies of denosumab [22-24, 27], with no new safety signals identified.

For the persistence analysis, an 8-week permissible gap was selected in line with previous studies in which gaps of 30, 60, and 90 days were used [5]. The choice of 8 weeks allowed for sensitivity analyses with 4 - to 12 -week windows, as used in a previous study [27]. In addition, these windows allowed for time taken by patients to make an appointment and secure prior authorization, if required, when their deadline for the next injection had arrived. The definition for adherence of 6 months \pm 4 weeks has been used in previous trials of denosumab conducted in patients with PMO [27-30] and is based on pharmacokinetic/pharmacodynamic data suggesting that residual effects of denosumab are evident up to 6 months after dosing [28]. Pharmacological coverage for 6 months over a 7month period equates to an MCR of $85 \%$, which is comparable with the $80 \%$ threshold of MPR for the definition of compliance [31, 32]. Indeed, the proportion of days that patients were covered by denosumab was high in these interim analyses, with an MCR of over $90 \%$ observed across the four countries.

While the characteristics of patients initiating denosumab were broadly similar across the countries, there were some notable differences. For example, in Greece, patients tended to be younger than in Germany, Austria, and Belgium, and the most common physician specialty varied by country. Although the mean age of patients was similar in Austria, Greece, and Belgium, the prevalence of prior osteoporotic fracture was substantially higher in Germany than in the other countries, which could reflect the use of country-specific guidelines regarding which patients are eligible for treatment [33-36]. Variations in patient characteristics could also be due to differences in healthcare systems, for example, the proportions of patients using private versus public clinical centers, or 
variations in reimbursement criteria for laboratory tests. The level of reimbursement of osteoporosis treatments also varies across the countries. Whereas in Austria and Germany these agents are fully reimbursed [37], in Greece patients pay 10$25 \%$ of the healthcare costs [38] and in Belgium patients pay $16-27 \%$ [39]. Owing to the differences observed in the baseline demographics of patients across the countries, no overall analyses were performed.

Despite the high proportion of patients who had received prior PMO treatment (81.3-88.8 \%), mean total hip, femoral neck, and lumbar spine T-scores were in or below the osteopenic range $(<-1.0)$. This, combined with the treating physicians' choices to change PMO therapy to denosumab, suggests that some physicians deemed prior medications to have failed. Alternatively, patients may have requested treatment change because of inconvenient dosing regimens or unacceptable tolerability. Furthermore, the patient-reported MMAS-8 scores indicate that the majority of patients had a low or medium score for adherence to prior medications [26]. Poor persistence with and adherence to other PMO medications are common $[32,40,41]$ and are associated with suboptimal fracture risk reduction [2-6]. Despite many of the patients in this study having a history of low adherence to medications, their persistence with and adherence to denosumab were high. Furthermore, in a univariate analysis performed to identify factors that were associated with 12-month persistence, MMAS- 8 scores at baseline did not influence persistence with denosumab. These observations suggest that the convenience of a subcutaneous 6-monthly dosing schedule may have influenced persistence, even among patients with a history of poor medication-taking behavior.

The pattern of higher persistence in patients with a DXA measurement compared with those without at least one Tscore measurement, observed in Austria and Greece, is of interest. Given the asymptomatic nature of osteoporosis, measuring BMD may provide patients with a clinically meaningful and informative tool with which they can define and monitor their disease. Patients may in turn be more informed about the nature and severity of osteoporosis, as well as the possible treatment options, and may subsequently be more likely to persist with medication. The absence of a DXA measurement may reflect the limited access to DXA machines or reimbursement restrictions, which in turn may also affect persistence.

As noted above previously, a univariate analysis was performed to identify factors associated with 12-month persistence. Owing to the high persistence in this study, it was difficult to identify factors that influenced persistence. No clinically relevant covariates were found to be significantly associated with persistence across all four countries, which could reflect differences in healthcare systems in the individual countries. However, for some covariates, similar nonsignificant trends were observed across the countries: increasing age, falls, living far from the clinic, and immobility were all associated with lower persistence. It may be that older or more frail patients are not able to visit their doctor as readily as others; hence, new formulations of LAIs may be advantageous in the treatment of elderly or less mobile patients as they may allow drug administration outside of the treating clinic by an individual adequately trained in injection techniques [21]. Patients with a parental history of hip fracture had increased persistence compared with those without such a history. This was a consistent trend across all four countries and may suggest that individuals who have observed the discomfort experienced by a close relative with an osteoporotic fracture may have higher motivation to persist with their own medication. Importantly, the number of comorbidities at baseline did not affect persistence, suggesting that denosumab is suitable for a wide range of patients. Participation in Prolong (an active support program whereby patients were sent reminders via phone calls, e-mails, and letters) significantly improved persistence in Austria, but not in the other countries in the study. This likely reflects the lack of availability of this program in Germany, Greece, and Belgium. Despite the majority of patients in Germany, Austria, and Belgium having access to other active or passive reminder programs, the univariate analysis found that the availability of such programs did not significantly affect persistence. This could reflect the high level of overall persistence with denosumab, rather than a lack of effectiveness for these initiatives.

The study had some limitations. Physicians actively chose to participate in this study, and their practices had the infrastructure to do so; therefore, they may not be representative of all physicians and sites treating women with PMO in Germany, Austria, Greece, and Belgium. Similarly, the patients in this study may not have been representative of the overall PMO population in the four countries. These patients were willing to participate and be monitored in a research study and were aware that their medication-taking behavior was being observed. As discussed previously, the individuals enrolled in this study may represent those with the most severe disease, owing to the high proportion of patients who had previously received another osteoporosis medication. Furthermore, the physicians participating in the study began enrolling patients approximately 1 year following the availability of denosumab in Europe and may have been early users of the product, potentially selecting denosumab as a treatment for those with chronic or severe disease; this could have biased the medication-taking behavior in either direction. In addition, the observational study design does not allow the impact of persistence on fracture risk to be evaluated. With regard to the univariate analysis of persistence, and as noted previously, it was difficult to identify any covariates that could be used in clinical practice to improve persistence further. No mathematical correction was made for multiple comparisons, and the univariate analysis did not consider the interaction of covariates; hence, a multivariate analysis is planned at the 24-month 
time-point. Finally, this study did not assess the number of patients who refused osteoporosis medication at first prescription (primary non-adherence). This was outside the scope of our study, but is nonetheless an important aspect of medication-taking behavior.

In spite of these limitations, this prospective, observational, multicenter study included a large range of medical center types, physicians, and patients across four European countries. Every attempt was made to avoid deviation from real-world practice in the study design, which should minimize confounding of the data. The decision to prescribe denosumab was made independently of, and prior to, enrollment in the study. Hence, there was no incentive for participating physicians to switch to denosumab, which may otherwise have influenced study outcomes. In a randomized, open-label study of bisphosphonate treatment persistence, closely aligning the study design with real-life practice resulted in persistence rates comparable to those from clinical records [42]. Therefore, the design of our study is likely to provide insight into the use of denosumab in real-life practice. In our study, the proportion of patients from each country who were persistent with denosumab at 12 months was higher than previously reported in observational studies of oral bisphosphonates $[2,16,17]$ or denosumab [43]. In addition, persistence levels were comparable to, or higher than, those observed in a randomized, clinical crossover trial of denosumab and alendronate [44]. Furthermore, data from an analogous, single-arm, prospective, observational study in the USA and Canada showed similar levels of persistence with denosumab (82\%) [27]. Together, these observations indicate that persistence with denosumab in real-world practice is similar to, or higher than, that observed in clinical studies.

In conclusion, the data reported here show that persistence, adherence, and MCR in the first 12 months of treatment were high in patients receiving denosumab in routine practice in Germany, Austria, Greece, and Belgium. The baseline characteristics of the patients in this study were typical of the general PMO population; therefore, it is likely that these data may be relevant to a wider patient group. Moreover, 12-month persistence with denosumab was 1.5-10-fold higher than that reported previously for bisphosphonates. Such increased persistence has the potential to improve patient outcomes, including fracture risk, in clinical practice, thereby reducing the rate of osteoporotic fracture and the associated burden on healthcare systems in Europe. A better understanding of patient and physician factors that influence medication-taking behaviors may help to improve persistence further.

Acknowledgments The authors thank the study centers, the women, and the investigators for their participation in this study. The authors would like to acknowledge Claire Desborough MSc of Amgen (Europe) $\mathrm{GmbH}$ and Kim Allcott $\mathrm{PhD}$ and Elizabeth Hartfield $\mathrm{PhD}$ of Oxford PharmaGenesis (UK), who provided editorial support. Funding for this support was provided by Amgen (Europe) $\mathrm{GmbH}$.
Funding This study was sponsored by Amgen Inc.

Conflicts of interest $\mathrm{PH}$ has received honoraria and research funding from Amgen, Roche, GlaxoSmithKline, Novartis, Merck Sharp and Dohme, Daiichi Sankyo, Pfizer, Procter and Gamble, Eli Lilly, and Nycomed.

NP has received research grants, consulting fees, and honoraria from Amgen, Eli Lilly, and Servier Laboratories.

EG has received lecture fees from Amgen, Novartis, and Servier.

MFT and LK-K are Amgen employees and shareholders.

EZ and GM were Amgen employees and shareholders at the time of conducting this study and during manuscript preparation.

IF has received research funding from Amgen and Eli Lilly.

PM has received lecture fees from Amgen, Pfizer, Leo Pharma, Genesis Pharma, Elpen, Vianex, and UniPharma and research grants from Amgen.

PG has received honoraria and research funding from Amgen, Roche, GlaxoSmithKline, Novartis, Merck Sharp and Dohme, Pfizer, Procter and Gamble, Eli Lilly, and Abbott.

HR has received speaker honoraria from Amgen, Novartis, Servier, and Eli Lilly.

AF-P is a principal investigator in studies sponsored by Amgen, Eli Lilly, and Servier Laboratories; has received unrestricted grants from Eli Lilly, Pfizer, and Roche; and has participated in speaker boards for Amgen, Daiichi Sankyo, Eli Lilly, GlaxoSmithKline, Merck Sharp and Dohme, Novartis, Roche, and Servier Laboratories.

Ethics All sites obtained local approval in accordance with the ethical principles of the Declaration of Helsinki. Each site provided Independent Ethics Committee or Independent Review Board protocol approval to Amgen along with all documentation pertaining to each patient before being permitted to participate in the study. All patients were assured of their right to withdraw from the study at any time without prejudice.

Open Access This article is distributed under the terms of the Creative Commons Attribution-NonCommercial 4.0 International License (http:// creativecommons.org/licenses/by-nc/4.0/), which permits any noncommercial use, distribution, and reproduction in any medium, provided you give appropriate credit to the original author(s) and the source, provide a link to the Creative Commons license, and indicate if changes were made.

\section{References}

1. Cramer JA, Roy A, Burrell A, Fairchild CJ, Fuldeore MJ, Ollendorf DA, Wong PK (2008) Medication compliance and persistence: terminology and definitions. Value Health 11:44-47

2. Hadji P, Claus V, Ziller V, Intorcia M, Kostev K, Steinle T (2012) GRAND: the German retrospective cohort analysis on compliance and persistence and the associated risk of fractures in osteoporotic women treated with oral bisphosphonates. Osteoporos Int 23:223-231

3. Hoer A, Seidlitz C, Gothe H, Schiffhorst G, Olson M, Hadji P, Haussler B (2009) Influence on persistence and adherence with oral bisphosphonates on fracture rates in osteoporosis. Patient Prefer Adherence 3:25-30

4. Huybrechts KF, Ishak KJ, Caro JJ (2006) Assessment of compliance with osteoporosis treatment and its consequences in a managed care population. Bone 38:922-928

5. Siris ES, Selby PL, Saag KG, Borgstrom F, Herings RM, Silverman SL (2009) Impact of osteoporosis treatment adherence on fracture rates in North America and Europe. Am J Med 122:S3-S13 
6. Lakatos P, Tóth E, Lang Z, Psachoulia E, Intorcia M (2013) Compliance protects against fracture in women with postmenopausal osteoporosis in Hungary. Value Health 16:A567

7. van den Boogaard $\mathrm{CH}$, Breekveldt-Postma NS, Borggreve SE, Goettsch WG, Herings RM (2006) Persistent bisphosphonate use and the risk of osteoporotic fractures in clinical practice: a database analysis study. Curr Med Res Opin 22:1757-1764

8. Netelenbos JC, Geusens PP, Ypma G, Buijs SJ (2011) Adherence and profile of non-persistence in patients treated for osteoporosisa large-scale, long-term retrospective study in The Netherlands. Osteoporos Int 22:1537-1546

9. Sambrook P, Cooper C (2006) Osteoporosis. Lancet 367:2010 2018

10. Downey TW, Foltz SH, Boccuzzi SJ, Omar MA, Kahler KH (2006) Adherence and persistence associated with the pharmacologic treatment of osteoporosis in a managed care setting. South Med J 99: 570-575

11. Hadji P, Minne H, Pfeifer M, Bourgeois P, Fardellone P, Licata A, Devas V, Masanauskaite D, Barrett-Connor E (2008) Treatment preference for monthly oral ibandronate and weekly oral alendronate in women with postmenopausal osteoporosis: a randomized, crossover study (BALTO II). Joint Bone Spine 75:303310

12. Kushida K, Shiraki M, Nakamura T, Kishimoto H, Morii H, Yamamoto K, Kaneda K, Fukunaga M, Inoue T, Nakashima M et al (2004) Alendronate reduced vertebral fracture risk in postmenopausal Japanese women with osteoporosis: a 3-year follow-up study. J Bone Miner Metab 22:462-468

13. Lee YH, Song GG (2011) Efficacy and safety of monthly $150 \mathrm{mg}$ oral ibandronate in women with postmenopausal osteoporosis: a systematic review and meta-analysis of randomized controlled trials. Korean J Intern Med 26:340-347

14. Cramer JA, Amonkar MM, Hebborn A, Altman R (2005) Compliance and persistence with bisphosphonate dosing regimens among women with postmenopausal osteoporosis. Curr Med Res Opin 21:1453-1460

15. Hadji P, Felsenberg D, Amling M, Hofbauer LC, Kandenwein JA, Kurth A (2014) The non-interventional BonViva Intravenous Versus Alendronate (VIVA) study: real-world adherence and persistence to medication, efficacy, and safety, in patients with postmenopausal osteoporosis. Osteoporos Int 25:339-347

16. Hadji PKS, Häussler B, Kless T, Linder R, Rowinski-Jablokow M, Verheyen F, Gothe H (2013) The bone evaluation study (BEST): patient care and persistence to treatment of osteoporosis in Germany. Int J Clin Pharmacol Ther 51:868-872

17. Lakatos P, Tóth E, Cina Z, Lang Z, Psachoulia E, Intorcia M (2013) Persistence \& compliance to treatment for osteoporosis in postmenopausal women in Hungary: a retrospective cohort study. Value Health 16:A567-A568

18. Hadji P, Ziller V, Gamerdinger D, Spieler W, Articus K, Baier M, Moericke R, Kann PH (2012) Quality of life and health status with zoledronic acid and generic alendronate - a secondary analysis of the Rapid Onset and Sustained Efficacy (ROSE) study in postmenopausal women with low bone mass. Osteoporos Int 23:2043-2051

19. Lee S, Glendenning P, Inderjeeth CA (2011) Efficacy, side effects and route of administration are more important than frequency of dosing of anti-osteoporosis treatments in determining patient adherence: a critical review of published articles from 1970 to 2009. Osteoporos Int 22:741-753

20. Boyle WJ, Simonet WS, Lacey DL (2003) Osteoclast differentiation and activation. Nature 423:337-342

21. Amgen (2014) Prolia ${ }^{\circledR}$ EU Summary of Product Characteristics [Available at: https://www.medicinesorguk/ema/medicine/23127]. Accessed November 2014

22. Brown JP, Prince RL, Deal C, Recker RR, Kiel DP, de Gregorio LH, Hadji P, Hofbauer LC, Alvaro-Gracia JM, Wang H et al (2009)
Comparison of the effect of denosumab and alendronate on BMD and biochemical markers of bone turnover in postmenopausal women with low bone mass: a randomized, blinded, phase 3 trial. J Bone Miner Res 24:153-161

23. Kendler DL, Roux C, Benhamou CL, Brown JP, Lillestol M, Siddhanti S, Man HS, San Martin J, Bone HG (2010) Effects of denosumab on bone mineral density and bone turnover in postmenopausal women transitioning from alendronate therapy. J Bone Miner Res 25:72-81

24. Cummings SR, San Martin J, McClung MR, Siris ES, Eastell R, Reid IR, Delmas P, Zoog HB, Austin M, Wang A et al (2009) Denosumab for prevention of fractures in postmenopausal women with osteoporosis. N Engl J Med 361:756-765

25. Lekkerkerker F, Kanis JA, Alsayed N, Bouvenot G, Burlet N, Cahall D, Chines A, Delmas P, Dreiser RL, Ethgen D et al (2007) Adherence to treatment of osteoporosis: a need for study. Osteoporos Int 18:1311-1317

26. Morisky DE, Ang A, Krousel-Wood M, Ward HJ (2008) Predictive validity of a medication adherence measure in an outpatient setting. J Clin Hypertens 10:348-354

27. Silverman SL, Siris E, Kendler DL, Belazi D, Brown JP, Gold DT, Lewiecki EM, Papaioannou A, Simonelli C, Ferreira I et al (2014) Persistence at 12 months with denosumab in postmenopausal women with osteoporosis: interim results from a prospective observational study. Osteoporos Int. doi:10.1007/s00198-014-2871-6

28. Bone HG, Bolognese MA, Yuen CK, Kendler DL, Wang H, Liu Y, San Martin J (2008) Effects of denosumab on bone mineral density and bone turnover in postmenopausal women. J Clin Endocrinol Metab 93:2149-2157

29. Genant HK, Engelke K, Hanley DA, Brown JP, Omizo M, Bone HG, Kivitz AJ, Fuerst T, Wang H, Austin M et al (2010) Denosumab improves density and strength parameters as measured by QCT of the radius in postmenopausal women with low bone mineral density. Bone 47:131-139

30. McClung MR, Lewiecki EM, Cohen SB, Bolognese MA, Woodson GC, Moffett AH, Peacock M, Miller PD, Lederman SN, Chesnut $\mathrm{CH}$ et al (2006) Denosumab in postmenopausal women with low bone mineral density. N Engl J Med 354:821-831

31. Freemantle N, Cooper C, Diez-Perez A, Gitlin M, Radcliffe H, Shepherd S, Roux C (2012) Results of indirect and mixed treatment comparison of fracture efficacy for osteoporosis treatments: a metaanalysis. Osteoporos Int 24:209-217

32. Kothawala P, Badamgarav E, Ryu S, Miller RM, Halbert RJ (2007) Systematic review and meta-analysis of real-world adherence to drug therapy for osteoporosis. Mayo Clin Proc 82: 1493-1501

33. Compston J, Bowring C, Cooper A, Cooper C, Davies C, Francis R, Kanis JA, Marsh D, McCloskey EV, Reid DM et al (2013) Diagnosis and management of osteoporosis in postmenopausal women and older men in the UK: National Osteoporosis Guideline Group (NOGG) update 2013. Maturitas 75:392-396

34. Dachverband Osteologie (2011) DVO guideline 2009 for prevention, diagnosis and therapy of osteoporosis in adults. Osteologie 20: 55-74

35. Kanis JA, McCloskey EV, Johansson H, Cooper C, Rizzoli R, Reginster JY (2013) European guidance for the diagnosis and management of osteoporosis in postmenopausal women. Osteoporos Int 24:23-57

36. Makras P, Vaiopoulos G, Lyritis GP (2012) 2011 guidelines for the diagnosis and treatment of osteoporosis in Greece. J Musculoskelet Neuronal Interact 12:38-42

37. Hernlund E, Svedbom A, Ivergård M, Compston J, Cooper J, Stenmark EV, McCloskey EV, Jönsson B, Kanis JA (2013) Osteoporosis in the European Union: medical management, epidemiology and economic burden. Arch Osteoporos 8:136 
38. ISPOR (2008) ISPOR global health care systems road map: Greece [http://www.ispor.org/htaroadmaps/Greece.asp]. Accessed March 2015

39. Institut national d'assurance maladie-invalidité (2014) Liste des spécialités pharmaceutiques remboursables : les catégories de remboursement [http://www.inami.fgov.be/fr/themes/coutremboursement/par-mutualite/medicament-produits-sante/ remboursement/specialites/Pages/liste-specialitespharmaceutiques-remboursables-categories-remboursement.aspx\#. VRExb8JybGg]. Accessed March 2015

40. Imaz I, Zegarra P, Gonzalez-Enriquez J, Rubio B, Alcazar R, Amate JM (2010) Poor bisphosphonate adherence for treatment of osteoporosis increases fracture risk: systematic review and meta-analysis. Osteoporos Int 21:1943-1951
41. Tosteson AN, Grove MR, Hammond CS, Moncur MM, Ray GT, Hebert GM, Pressman AR, Ettinger B (2003) Early discontinuation of treatment for osteoporosis. Am J Med 115:209-216

42. Cooper A, Drake J, Brankin E (2006) Treatment persistence with oncemonthly ibandronate and patient support vs. once-weekly alendronate: results from the PERSIST study. Int J Clin Pract 60:896-905

43. Karlsson L, Lundkvist J, Intorcia M, Psachoulia E, Strom O (2013) Treatment persistence in Swedish women initiating denosumab treatment for postmenopausal osteoporosis. Value Health 16:A567

44. Freemantle N, Satram-Hoang S, Tang ET, Kaur P, Macarios D, Siddhanti S, Borenstein J, Kendler DL (2012) Final results of the DAPS (Denosumab Adherence Preference Satisfaction) study: a 24-month, randomized, crossover comparison with alendronate in postmenopausal women. Osteoporos Int 23:317-326 